\title{
Queere Jugendliche in der stationären Erziehungshilfe
}

\author{
Biographische Perspektiven auf Heteronormativität
}

In Biographien von Jugendlichen, die in der stationären Erziehungshilfe leben, werden Verhältnisse von Heteronormativität sichtbar. Welche Anforderungen mit Normierungen und Hierarchisierungen entlang heteronormativer Vorstellungen aus einer biographischen Perspektive verbunden sein können, wird im Folgenden skizziert. Von diesen Anforderungen ausgehend werden Schlussfolgerungen für eine queergerechte Unterstützung und Begleitung von Kindern und Jugendlichen skizziert.

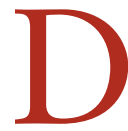
ie Unterstützung von Jugendlichen und ihren Familien steht im Zentrum von Angeboten der Hilfen zur Erziehung im deutschsprachigen Raum. Zum Thema Gender liegen im Bereich stationäre Erziehungshilfen Studien vor, die sich mit den Erfahrungen von bspw. Mädchen in der stationären Erziehungshilfe beschäftigen (bspw. Finkel 2004; Zeller 2012). Im fachlichen Diskurs in der stationären Erziehungshilfe spielt das Thema der sexuellen und geschlechtlichen Vielfalt bislang so gut wie keine Rolle. Im pädagogi-

Angela Rein
FHNW University of Applied Sciences and Arts Northwestern
Switzerland, Muttenz, Schweiz
*1979; Dr., Diplom-Pädagogin, Professorin am Institut Kinder-
und Jugendhilfe der Hochschule für Soziale Arbeit, Fachhoch-
schule Nordwestschweiz (FHNW).
angela.rein@fhnw.ch

Zusammenfassung In den Biographien von queeren Jugendlichen in der stationären Erziehungshilfe werden Ausgrenzungserfahrungen sichtbar. Heteronormative Normalitätskonstruktionen führen dazu, dass LGBTIQ*-Jugendliche in ihrem Alltag zu Anderen gemacht werden. Heteronormativität in der stationären Erziehungshilfe kann sich bspw. in Familienkonstruktionen ausdrücken oder im Unsichtbarmachen von queeren Lebensweisen. In den Biographien von queeren Jugendlichen zeigen sich ambivalente Umgangsstrategien damit. Der Beitrag gibt Anregungen für eine queer-gerechte Jugendhilfe, die Normalitätskonstruktionen und hegemoniale Ordnungen in der stationären Erziehungshilfe hinterfragen.

Schlüsselwörter Normalitätskonstruktionen, Kinder- und Jugendhilfe, Stationäre Erziehungshilfe, Heteronormativität, Dekonstruktion, Queere Jugendliche, LGBTIQ*, Vulnerabilität, Subjektivierung, Biographie schen Alltag hingegen bestehen Unsicherheiten, wenn sich bspw. ein Trans-Junge in der Mädchenwohngruppe outet und dadurch die Frage aufgeworfen wird, welche Anforderungen daraus für die pädagogische Begleitung folgen.

\section{Heteronormative Ordnungen}

in der stationären Erziehungshilfe?

Gesellschaftliche Ordnungen und Differenzverhältnisse spielen in der pädagogischen Begleitung eine zentrale Rolle. Verbindend bei gesellschaftlichen Ordnungen (wie bspw. Heterosexismus, Rassismus, Ableismus oder Klassismus) ist die Legitimation von Benachteiligung aufgrund sozialer Differenzen, in deren Folge Machtverhältnisse reproduziert werden (vgl. Kessl und Plößer 2010). So konstruieren etwa ableistische Ordnungen (Ability (engl.) = Fähigkeiten) Normen, in denen Vorstellungen von Fähigkeiten und Handlungsfähigkeit festgeschrieben werden und Menschen vor dem Hintergrund dieser Folie bewertet werden (Köbsell 2015). Für die Institution der Schule liegen Untersuchungen vor, die aufzeigen, wie heteronormative (Kleiner 2015), rassistische (Rose 2012) oder ableistische (Buchner 2018) Ordnungen Jugendliche subjektivieren und diskriminieren. Für die stationäre Kinder- und Jugendhilfe sind ähnliche Prozesse anzunehmen. Heteronormativität als gesellschaftliche Ordnung bringt dabei normative Vorstellungen mit sich, die davon ausgehen, dass es zwei klar voneinander abgrenzbare Geschlechter gibt, die bei der Geburt eindeutig zugewiesen werden können. Weiterhin gehört dazu die Normalitätsvorstellung, dass sexuelle und romantische Anziehung jeweils vom „anderen“ Geschlecht ausgeht und auf zwei Personen beschränkt ist (Hartmann 2012, S. 153 ff.). 


\section{Extrablick: Kinder- und Jugendhilfe und LGBTIQ*}

In Prozessen der Subjektivierung werden mit Butler Personen durch Adressierungen (bspw. als lesbisch, trans* oder männlich") erst zu „Subjekten gemacht“. Subjekte werden also im Sozialen hervorgebracht. Hierbei sind die Verletzbarkeiten auf der Ebene von Körpern, Identität und Subjektivität, gesellschaftliche Anerkennungsmöglichkeiten oder auch positive Formen der Selbstbezugnahme ungleich verteilt: „Verleihen also bestimmte Arten der Anrufung Identität, dann konstituieren die verletzenden Anrufungen durch Verletzung Identität“ (Butler 2001, S. 100). Wie Jugendhilfe ihre Adressat_innen subjektiviert und wie hier Heteronormativität als symbolische Ordnung Verletzbarkeit hervorbringen kann wird im Folgenden skizziert.

\section{Biographische Perspektiven auf Erziehungshilfe- erfahrungen}

Empirische Grundlage des Artikels ist meine Dissertationsstudie, die sich mit den Bedeutungen von Normalitätskonstruktionen im Übergang aus der stationären Erziehungshilfe aus einer biographischen Perspektive im Kontext von gesellschaftlichen Macht- und Ungleichheitsverhältnissen beschäftigt hat (Rein 2020). Befragt wurden Jugendliche und junge Erwachsene in der Schweiz, die entweder kurz vor dem Übergang aus der stationären Erziehungshilfe stehen oder diese bereits bis zu zehn Jahre verlassen haben. Auffallend in den biographischen Erzählungen ist, dass diese Erklärungen geben, wie es dazu gekommen ist, dass die jungen Menschen in die stationäre Erziehungshilfe mussten. Die Studie macht deutlich, dass der Übergang in die stationäre Erziehungshilfe sehr stark ent-normalisierend erlebt wird. Queere Jugendliche sind dabei in der Jugendhilfe zusätzlich heteronormativen Ordnungen ausgesetzt, die deren potenzielle Verletzbarkeit im Kontext der Unterstützung verstärken kann.

\section{Alltäglich Ausgrenzungserfahrungen}

Erfahrungen der Ausgrenzung werden von den Kindern und Jugendlichen insbesondere in Institutionen der Schule, aber auch der stationären Erziehungshilfe, beschrieben. Marla Brunori kommt im Alter von 15 Jahren in die stationäre Erziehungshilfe auf eine Mädchenwohngruppe. Als Begründungen für ihren Heimaufenthalt nennt Marla Bruonori Probleme zu Hause nach der Trennung ihrer Eltern, psychische Belastungen und selbstverletzendes Verhalten. Diese verschiedenen Themen führen zu einer Krise, in der alles „den Bach runter gegangen ist“. In der Folge geht sie in eine stationäre Einrichtung.

Parallel dazu beschreibt Marla Brunori die Schule als einen heteronormativen Ort, an dem sie auf der PeerEbene Ausgrenzungen erfährt, weil sie lesbisch ist. Sie outet sich im Alter von 14 Jahren in der Schule, als sie sich das erste Mal in ein Mädchen verliebt:

M: (1) ich hatte auch schlechte Erfabrungen in der Schule als ich mich das erste Mal geoutet habe und das war eine Katastrophe und (3) und [...] Die haben dann gesagt ja wir können nicht mehr mit dir in die Pause gehen weil äh die haben Angst fü-we-wir sind jetzt lesbisch und (.) ich hatte dann auch richtig Angst auf die Mädchen zuzugehen weil die sonst denken ja ich will die heiraten oder was weiß ich ja

Hier wird deutlich, wie ihr auf dem Schulhof in der Pause von den anderen vermittelt wird, dass ihr sexuelles Begehren nicht der Norm entspricht. Sie wird zu einer gemacht, vor der die anderen „Angst“ haben, da sich die anderen gleichaltrigen Mädchen durch den Kontakt mit ihr offensichtlich in ihrer Normalität bedroht sehen. Marla Brunori wird in der Folge mit ihrem sexuellen Begehren für alle sichtbar gemacht und besondert. Gleichzeitig wird sie als bedrohlich konstruiert. So werden Machtordnungen umgedeutet, und Marla Brunori wird eine potenzielle Übergriffigkeit zugesprochen, vor der sich die anderen vordergründig schützen. In der Folge wird sie ausgegrenzt und dabei verdeckt, mit welchen Folgen und mit welcher Verletzbarkeit diese Grenzziehungen auf dem Pausenhof und ihre dementsprechende Subjektposition als Andere, als Nicht-Normale verbunden sind.

Diese Alltäglichkeit von Ausgrenzungserfahrungen wiederholen sich in den Fällen von Jugendlichen, die queer leben in unterschiedlichen Kontexten wie im Peerkontext, in Beratungssettings, in der Familie oder auch in der stationären Erziehungshilfe. Marla Brunori ist zum Zeitpunkt des Interviews 24 Jahre alt und resümiert ihren Umgang mit Erfahrungen von Heteronormativität so:

M: ich hatte lange Probleme ich habe auch jetzt noch ich bin nicht ganz überall geoutet also im Geschäft das wissen schon Einzelne aber (1) sondern ich ich (.) pu(.) ich ge- renne da jetzt nicht ins Geschäft und sage ja ich bin ich stehe auf Franen und bin mit einer Frau zusammen

Hier zeigt sich, dass für sie eine Strategie ist, ihr Begehren zu verdecken und sie sich auf diese Art vor potenziellen Verletzungen schützt. In Bezug auf LeavingCare-Prozesse, also den Übergangen aus stationären Einrichtungen ins Erwachsenenalter, wird die Vulnerabilität von queeren Care Leavern durch Erfahrungen von Heteronormativität verstärkt. So kommt zur 
Othering-Erfahrung, nicht normal, sondern im Heim aufgewachsen zu sein, die Zuschreibung queer und dadurch „anders“ zu sein.

\section{Subtile Praxen der Besonderung in der stationären Erziehungshilfe}

Die Verletzbarkeit aufgrund einer Subjektposition, die als „sexuell abweichend" markiert ist, spielt auch bei Liah Petrovic in den Peerkontakten in der stationären Erziehungshilfe eine Rolle. Liah Petrovic fühlt sich in der Mädchenwohngruppe am Anfang wegen ihrer sexuellen Orientierung nicht wohl:

L: (2) ganz am Anfang ist schwierig gewesen für mich weil die (.) alle gewusst haben (.) ich habe eine Freundin (.) und dann hat es zuerst so (1) hmm (.) so Spannungen gegeben, (.) wegen dem obwohl ich (.) niemandem irgendwie etwas gemacht habe, (2) bis die mich dann halt kennengelernt haben ja: (.) und dann ist (.) alles gut gewesen (.)

Liah Petrovic nennt „Spannungen“ im Übergang in die Wohngruppe der stationären Erziehungshilfe, weil sie eine lesbische Beziehung hat und dies den anderen Mädchen bekannt wird. Aufgrund ihrer Beziehung zu einer Frau macht sie Ausgrenzungserfahrungen, die allerdings, wie sie an einer anderen Stelle beschreibt, sehr subtil gewesen seien:

\section{L: Ab das sind so (.) die all die Sachen wo eben (2) nicht} so offensichtlich sind (.)das sind so Blick, das sind so (.) ((pff)) und äh (.)wie halt (.) das (.) das ist alles so binten rum (.)zwischen den Zeilen (1) ich habe aber nie ein Problem gehabt mit dem (.)

Auffallend in dieser Darstellung ist ihre Unterstreichung, dass sie mit den subtilen Praxen der Besonderung aber "nie ein Problem gehabt" habe. Dies kann auch daraufhin deuten, dass die Erfahrungen für sie alltäglich sind und sie damit einen Umgang entwickelt hat oder auf die Strategie, die eigene Verletzbarkeit zu verdecken und sich selbst handlungsfähig zu fühlen. Gleichzeitig sind diese Prozesse der Ausgrenzung auf Peer-Ebene offensichtlich ohne institutionelle Intervention möglich.

Sichtbar ist, dass zu Beginn in der stationären Erziehungshilfe ihre selbstverständliche Zugehörigkeit infrage gestellt wird. Ihr sexuelles Begehren wird in unterschiedlichen Kontexten als nicht normal konstruiert. Es werden Adressierungen und Ausgrenzungen in den gesellschaftlichen Institutionen der stationären Erziehungshilfe gemacht, die ihre Normalität aufgrund der sexuellen Orientierung infrage stellen und Zugehörigkeiten zu den anderen Peers zumindest in der Anfangsphase erschweren. Aus biographischer Perspektive knüpfen diese Erfahrungen an eine Reihe an Erfahrungen an, die Liah im Kontext von Schule gemacht hat. Verschiedene Dominanz- und Unterdrückungsordnungen überlagern sich hier in den Institutionen Schule und stationäre Erziehungshilfe, wobei die Ebene der Peers aus der Perspektive der jungen Menschen bei der Reproduktion dieser Ordnungen eine zentrale Rolle spielt. Bettina Kleiner (2015) hat die schulischen Differenzerfahrungen lesbischer, schwuler, bisexueller und Trans"Jugendlicher untersucht. Sie verweist dabei auf die Verbindung von direkten verletzenden Anrufungen durch andere Schüler_innen mit der fehlenden Anerkennung von queeren Lebensweisen in der Institution der Schule (vgl. Kleiner 2015, S. 327 ff.). Somit weisen also die Praxen der Jugendlichen immer auf Normalitätsordnungen hin, auf die sich die Peers in ihren verletzenden Anrufungen berufen. So ist es notwendig zu markieren, dass es verkürzt wäre, die beschriebenen Praxen isoliert zu betrachten und die Ebene der Peers als den Ort zu markieren, an dem diese Ausgrenzungen entstehen. Vielmehr greifen die Biograph_innen und ihre Peers auf hegemoniale Normalitätsvorstellungen zurück, wobei Prozesse des Positioniert-Werdens und des Sich-selbstPositionierens stattfinden.

Die hier in den Interviews beschrieben subtilen Praxen und Witze auf Peer-Ebene werden im Kontext der stationären Erziehungshilfe gebilligt und nicht sanktioniert. So zeigt sich in der vorliegenden Untersuchung, dass die Notwendigkeit einer Mädchen-Wohngruppe konzeptionell so begründet wird, dass der Raum ohne männliche Jugendliche frei von Sexualität wäre und den Mädchen andere Entfaltungsmöglichkeiten bieten würde. Dies verweist darauf, dass queere Lebensweisen unsichtbar gemacht und de-thematisiert werden.

Die in den Interviews sichtbar werdenden heteronormativen Ausgrenzungserfahrungen machen sichtbar, dass für queere Jugendliche die Institution der stationären Erziehungshilfe nicht selbstverständlich ein sicherer Ort ist. Queere Jugendliche scheinen in stationären Einrichtungen nicht-normal bzw. unsichtbar gemacht zu werden. Ausgrenzungserfahrungen gehören zum Alltag und auch im Übergang ins Erwachsenenalter spielt Heteronormativität eine Rolle und die jungen Menschen sind herausgefordert damit Umgangsstrategien zu finden.

\section{Ambivalente Reproduktion von heteronormativen Familienvorstellungen}

Eine bedeutsame Arena für Aushandlungen rund um den Unterstützungsbedarf von Kindern, Jugendlichen 


\section{Sozial Extra}

\section{Extrablick: Kinder- und Jugendhilfe und LGBTIQ*}

sowie deren Eltern ist die Frage, inwieweit Eltern ihrer Sorgepflicht nachkommen können. Damit einher gehen auch normative Vorstellungen von Familie. Aus einer biographischen Perspektive zeigen sich hier Anstrengungen, die eigene Familiengeschichte als möglichst normal zu konstruieren. Dabei zeigt sich eine starke Bezugnahme zu heteronormativen Bildern von Familie. Auch Marla Brunori setzt sich in ihrem Interview stark mit normativen Bildern von Familie auseinander. Zum Zeitpunkt des Interviews lebt sie mit ihrer Freundin in der Einliegerwohnung der Eltern der Freundin. Das Paar plant von dort auszuziehen, sobald beide ihre Ausbildungen abgeschlossen haben. In ihrer Biographie konstruiert sie die Familie ihrer älteren Schwester als „Bilderbuchfamilie“. Dies verweist als Begriff auf eine idealisierte Vorstellung von Familie. Für sie geht damit einher, dass sie sich auf einer emotionalen Ebene gut aufgehoben fühlt und sich dort gerne aufhält. Was diese „Bilderbuchfamilie“ für sie auszeichnet, führt sie so aus:

M: Ja ich meine die haben ein Haus, ich meine sie haben die vier Kinder da ha-da wurde immer zusammen am Tische gegessen der Vater war dann irgendwann mal zurück von der Arbeit und (.) meine Schwester immer zu Hause

In dieser Beschreibung zeigen sich normative Bilder eines bürgerlichen heteronormativen Familienmodells, bei dem eine klassische Arbeitsteilung zwischen den Geschlechtern besteht und die Frau für die Kinder zu Hause und den Haushalt zuständig ist, der Mann hingegen der Erwerbsarbeit nachgeht. Mit der Eröffnung der Sequenz über den Raum der Familie - „die haben ein Haus " - wird die idealisierte Familie damit zugleich auch in einen klaren Klassenkontext gesetzt. Am Ende des Tages treffen sich alle Personen der Kernfamilie zum gemeinsamen Abendessen. Der Verweis darauf, dass „immer zusammen am Tische gegessen " wird, markiert eine Kultur des gemeinsamen Essens, was an eine von bürgerlichen Idealen geprägte Familienkultur erinnert.

Dieses heteronormative Lebensmodell stellt Marla in ihrem Interview nicht in Frage, auch wenn ihr eigener Lebensentwurf davon abweicht. Für ihren Lebensentwurf in der Zukunft scheint das Modell einer Familie nicht anwendbar. Vielmehr wird in der Erzählung sichtbar, dass sie mit ihrem Lebensmodell als lesbische Frau als Andere positioniert wird und sie hier nicht den gesellschaftlich dominanten Vorstellungen von Normalität entsprechen kann.

Auch andere Studien zu Familie in der stationären Erziehungshilfe machen sichtbar, dass dort heteronormative und bürgerliche Vorstellungen von Familie dominant sind (Pomey 2017). Dies führt auf einer biographischen Ebene dazu, dass eigene Erfahrungen von Familie, die diesen klassischen Vorstellungen nicht entsprechen, legitimiert werden müssen oder auch unsichtbar bleiben.

\section{Stationäre Angebote „ver-queeren"?}

In den biographischen Einblicken der queeren Jugendlichen wird sichtbar, dass Heteronormativität in der stationären Erziehungshilfe auf unterschiedlichen Ebenen sichtbar wird und die Jugendlichen so als Andere, als Nicht-Normale-Jugendliche markiert und positioniert werden. So reihen sich Erfahrungen in der stationären Erziehungshilfe aus biographischer Sicht an eine ganze Reihe an Erfahrungen in anderen Kontexten ein sei es mit Peers, in der Schule oder auch im familiären Umfeld.

Die Begleitung von queeren Kindern und Jugendlichen stellt daher insbesondere als Anforderung an die stationäre Erziehungshilfe, dominante Normalitätsvorstellungen zu „ver-queeren“, also zu hinterfragen, zu dekonstruieren und zu verändern.

Dekonstruktion meint die Beschäftigung mit den scheinbaren Natürlichkeiten und Selbstverständlichkeiten, die mit heteronormativen Vorstellungen einhergehen. Dies würde auch bedeuten, die Perspektiven auf Geschlechter und sexuelle Orientierungen in Bezug auf die Gestaltung der Räume zu erweitern. Welche Geschlechter werden mitbedacht und mitbenannt? Wer kann in die Wohngruppe für Mädchen*? Wie sprechen wir die Jugendlichen an? Auf welche Lebensentwürfe und Entwürfe von Familie bereiten wir die Jugendlichen im Heim vor? Was für Vorstellungen von Familie haben wir? Nehmen wir dabei queere Eltern wahr, oder werden diese mit unseren heteronormativen Vorstellungen unsichtbar gemacht? Die Fragen können anregen, die räumliche Gestaltung, die Sprache und konzeptionelle Perspektiven zu hinterfragen.

Mit einer ver-queerten Perspektive würde es also sehr stark darum gehen, die Wirkmächtigkeit gesellschaftlicher hegemonialer Ordnungen in der Gestaltung stationärer Erziehungshilfe kritisch zu hinterfragen und gerade an scheinbaren Selbstverständlichkeiten anzusetzen. Ver-queeren meint eine Haltung, die einen Blick auf die institutionellen Selbstverständlichkeiten einnimmt, die Barrieren bilden können und weniger, die Gruppe der queeren Jugendlichen als Andere ins Zentrum zu stellen, die besondere Bedarfe haben. Die Bedarfe hängen sehr viel mit Erfahrungen zusammen, nicht selbstverständlich anerkannt und normal sein zu können und auch im Alltag der stationären Erziehungshilfen ein Ausnahmefall zu sein, der Probleme mit sich bringt, weil bspw. auf einer Mädchenwohngruppe ein Trans-Mädchen sein 
will oder die Sexualität lesbischer Mädchen dort nicht mitgedacht wird.

Eingegangen. 11. Januar 2021

Angenommen. 21. Januar 2021

Funding. Open access funding provided by FHNW University of Applied Sciences and Arts Northwestern Switzerland.

Open Access. Dieser Artikel wird unter der Creative Commons Namensnennung 4.0 International Lizenz veröffentlicht, welche die Nutzung, Vervielfältigung, Bearbeitung, Verbreitung und Wiedergabe in jeglichem Medium und Format erlaubt, sofern Sie den/die ursprünglichen Autor(en) und die Quelle ordnungsgemäß nennen, einen Link zur Creative Commons Lizenz beifügen und angeben, $o b$ Änderungen vorgenommen wurden.

Die in diesem Artikel enthaltenen Bilder und sonstiges Drittmaterial unterliegen ebenfalls der genannten Creative Commons Lizenz, sofern sich aus der Abbildungslegende nichts anderes ergibt. Sofern das betreffende Material nicht unter der genannten Creative Commons Lizenz steht und die betreffende Handlung nicht nach gesetzlichen Vorschriften erlaubt ist, ist für die oben aufgeführten Weiterverwendungen des Materials die Einwilligung des jeweiligen Rechteinhabers einzuholen.

Weitere Details zur Lizenz entnehmen Sie bitte der Lizenzinformation auf http://creativecommons.org/licenses/by/4.0/deed.de

\section{Literatur}

Buchner, T. (2018). Die Subjekte der Integration. Schule, Biographie und Behinderung. Bad Heilbrunn: Klinkhardt.

Butler, J. (2001). Psyche der Macht. Das Subjekt der Unterwerfung. Frankfurt am Main: Suhrkamp.

Finkel, M. (2004). Selbstständigkeit und etwas Glück. Einflüsse öffentlicher Erziehung auf die biographischen Perspektiven junger Frauen. Weinheim, München: Juventa.

Hartmann, J. (2012). Improvisation im Rahmen des Zwangs. In N. Ricken \& N. Balzer (Hrsg.), Judith Butler: Pädagogische Lektüren (S. 149178). Wiesbaden: VS.

Kessl, F., \& Plößer, M. (2010). Differenzierung, Normalisierung, Andersheit. Soziale Arbeit als Arbeit mit den Anderen - eine Einleitung. In F. Kessl $\&$ M. Plößer (Hrsg.), Differenzierung, Normalisierung, Andersheit. Soziale Arbeit als Arbeit mit den Anderen (S. 7-16). Wiesbaden: VS.

Kleiner, B. (2015). subjekt bildung heteronormativität. Rekonstruktion schulischer Differenzerfahrungen lesbischer, schwuler, bisexueller und Trans"Jugendlicher. Opladen, Berlin, Toronto: Budrich.

Köbsell, S. (2015). Ableism: Neue Qualität oder „alter Wein“ in neuen Schläuchen? In I. Attia, S. Köbsell \& P. Nivedita (Hrsg.), Dominanzkultur reloaded: Neue Texte zu gesellschaftlichen Machtverhältnissen und ibren Wechselwirkungen (S. 21-34). Bielefeld: transcript.

Pomey, M. (2017). Vulnerabilität und Fremdunterbringung. Weinheim: Beltz Juventa.

Rein, A. (2020). Normalität und Subjektivierung. Eine biographische Untersuchung im Übergang aus der stationären Jugendhilfe. Bielefeld: transcript. https://www.transcript-verlag.de/978-3-8376-5170-6/normalitaet-und-subjektivierung/?number=978-3-8394-5170-0\&c=313000106

Rose, N. (2012). Migration als Bildungsherausforderung. Subjektivierung und Diskriminierung im Spiegel von Migrationsbiographien. Bielefeld: transcript.

Zeller, M. (2012). Bildungsprozesse von Mädchen in den Erziehungshilfen. Weinheim, Basel: Beltz Juventa. 